\title{
Substrate-Selective Chemical Vapor Deposition of Reactive Polymer Coatings**
}

\author{
By Hsien-Yeh Chen, Joseph Hermes Lai, Xuwei Jiang, and Joerg Lahann*
}

Rapid progress in biology and biotechnology ${ }^{[1-5]}$ has sparked a keen interest in micro- and nanostructured surfaces. A large number of strategies have been developed for the fabrication of surfaces that require a spatially controlled distribution of biomolecules, such as proteins, bioligands, polysaccharides, or polyethylene glycol (PEG). Existing approaches can grossly be categorized into processes relying on either physisorption or covalent immobilization. Covalent microstructuring methods include soft-lithographic patterning of monomers or initiators followed by surface-induced polymerization, ${ }^{[6,7]}$ photolithography, ${ }^{[8-10]}$ as well as spatially controlled masking or templating of surfaces. ${ }^{[11,12]}$ For applications, where defined biological structures have to be maintained over extended periods of time, covalent immobilization is typically considered superior. ${ }^{[13]}$ However, covalent coupling requires the presence of adequate functional groups on the substrate surface. Because the availability of chemical surface groups is defined by the chemical makeup of the substrate material itself, choice of coupling chemistries is often limited and covalent immobilization can involve rather complex reaction cascades. The ideal surface modification method will thus be able to decouple immobilization chemistry from the substrate composition. ${ }^{[14]}$ Decoupling the surfaces from the bulk material(s) not only expands the diversity of immobilization schemes that can be found for a given substrate, but also ensures that a proven immobilization method can easily be transferred from one substrate to another, without the need for repeated reconfiguration of the underlying surface chemistry. Ultrathin polymer coatings, which essentially function as a surface replacement, have been widely pursued in this respect. Examples include both solutionand vapor-based deposition methods. ${ }^{[15-20]}$

Based on chemical vapor deposition (CVD) polymerization of [2.2]paracyclophane derivatives, we have developed a series of functionalized poly- $p$-xylyenes with a wide range of different chemical groups for surface modification via covalent immo-

[*] H.-Y. Chen, J. H. Lai, X. Jiang, Prof. J. Lahann

Departments of Chemical Engineering, Materials Science and Engineering, and Macromolecular Science and Engineering University of Michigan

2300 Hayward Street, Ann Arbor, MI 48109 (USA)

E-mail: lahann@umich.edu

[**] J. L. gratefully acknowledges support from the NSF in form of a CAREER grant (DMR-0449462) and funding from the NSF under the MRI program (DMR 0420785). The project was further funded by an Idea Award of the Department of Defense. Supporting Information is available online from Wiley InterScience or from the authors. bilization. These reactive polymer coatings are close relatives of the nonreactive parylene coatings, but, in addition, enable covalent immobilization of proteins, ${ }^{[21,22]}$ sugars, ${ }^{[23]}$ or PEG. ${ }^{[24]}$ Depending on their chemical side groups, functionalized poly-p-xylylenes can be used for spatially controlled immobilization via microcontact printing ${ }^{[25,26]}$ photolithographic techniques, ${ }^{[12,24]}$ or polymer templating using vaporassisted microstructuring in replica structures (VAMPIR). ${ }^{[27]}$ While these methods rely on physical aids, such as stamps or masks, an even simpler approach for obtaining spatially controlled surface modification would be the selective inhibition of the CVD polymerization process due to differences in the substrate chemistry. Jensen and coworker reported selective inhibition of parylene $\mathrm{N}$, parylene $\mathrm{C}$ as well as poly ( $p$-phenylene vinylene) (PPV) by iron and iron salts, ${ }^{[28]}$ and used selective CVD polymerization to create a wide range of patterns. ${ }^{[28]}$ In an extension of this work, it was shown that several transitional metals, metal salts, and organometallic complexes similarly inhibited the growth of parylene $\mathrm{N}$ and parylene $C{ }^{[29]}$ Suh et al. have used selective CVD polymerization within sub-micrometer scale polydimethylsiloxane (PDMS) channels to yield high aspect-ratio structures. Surface-coated PDMS channels of as little as $180 \mathrm{~nm}$ in width were obtained by depositing iron on the bottom of microchannels and then selectively growing polymer on the channel sidewalls only. ${ }^{[30]}$ While selective inhibition has been well established for parylene $\mathrm{N}$ and $\mathrm{C}$, the question of whether this simple microstructuring concept can be extended to reactive polymer coatings, i.e., functionalized poly- $p$-xylylenes, remains unanswered.

In this Communication, we systematically investigated the selective inhibition of CVD polymerization by a series of metals and discovered the first metal-mediated inhibition of a functionalized poly- $p$-xylylene (poly[4-vinyl- $p$-xylylene-co- $p$ xylylene]). Reactivity of the vinyl groups via cross-metathesis reaction is demonstrated. The fact that this selectively deposited reactive coating is equipped with functional groups for further surface modification may provide an extremely simple access route towards micro- and nanostructured biointerfaces.

To assess the inhibitory character of metals on the CVD polymerization of substituted [2.2]paracyclophanes, a series of different poly- $p$-xylylenes were deposited. Taking advantage of previously established principles of CVD polymerization, ${ }^{[20,31-38]}$ we prepared four nonreactive and ten reactive poly- $p$-xylylenes containing a wide range of different functional groups such as amines, ${ }^{[9]}$ alcohols,${ }^{[40,41]}$ aldehydes, ${ }^{[23]}$ anhy- 
drides, ${ }^{[26]}$ esters, ${ }^{[21,25,42]}$ alkyne, ${ }^{[43]}$, or ketones. ${ }^{[27,44]}$ The CVD films were simultaneously deposited on a library of nine different metal surfaces: $\mathrm{Cu}, \mathrm{Ir}, \mathrm{Ta}, \mathrm{W}, \mathrm{Pt}, \mathrm{Ni}, \mathrm{Ti}, \mathrm{Ag}$, and $\mathrm{Au}$. These metals were selected based on previous use as inhibitor candidates ${ }^{[29]}$ and because they were readily available through sputtering or electron-beam (e-beam) deposition. After deposition, the metal surfaces were allowed to be in contact with atmospheric conditions during storage. For CVD polymerization, the metal/silicon substrates were placed onto a temperature-controlled stage $\left(15 \pm 5^{\circ} \mathrm{C}\right)$ inside of the CVD polymerization chamber. As shown in Figure 1c, substituted [2.2]paracyclophanes were first sublimed and the resulting dimers were transferred into the pyrolysis zone. After thermal conversion into the corresponding quinodimethanes, polymerization occurred on cooled samples placed in the deposition chamber. During CVD polymerization, polymer growth rates were estimated to be about $0.5 \AA \mathrm{As}^{-1}$ based on in situ quartz crystal microbalance (QCM) analysis. Figure 1a summarizes the inhibition behavior of nine different metals for both, nonfunctional (1-4) and reactive (5-14) poly- $p$-xylylenes. Film thicknesses were controlled in the range of $40-80 \mathrm{~nm}$ according to QCM and were also confirmed by ellipsometry after deposition. The chemical signatures of the metal surfaces after CVD polymerization were assessed using grazing angle Fourier- transformed infrared reflection adsorption spectroscopy (IRRAS). IRRAS is an extremely surface sensitive method and self-assembled monolayers on $\mathrm{Au}$ and $\mathrm{Ag}$ with less than $2 \mathrm{~nm}$ thickness are routinely studied with this method. ${ }^{[45-48]}$ Typical IRRAS spectra are shown in Figure 1b, which were recorded after CVD of poly(dichloro- $p$-xylylene) (3) on $\mathrm{Cu}, \mathrm{Ir}$, $\mathrm{Ta}, \mathrm{W}, \mathrm{Pt}, \mathrm{Ni}, \mathrm{Ti}, \mathrm{Ag}$, and $\mathrm{Au}$. The spectra are dominated by characteristic $\mathrm{C}-\mathrm{Cl}$ stretches at $1030-1100 \mathrm{~cm}^{-1}$, which were present on $\mathrm{Au}, \mathrm{Ni}, \mathrm{Pt}, \mathrm{W}$, and Ta surfaces. In contrast, these bands could not be observed on $\mathrm{Ag}, \mathrm{Cu}$, Ir, or Ti surfaces. Similarly, other characteristic signals were absent on the later group of metal surfaces, suggesting selective inhibition of the CVD process on $\mathrm{Ag}, \mathrm{Cu}$, Ir, or Ti surfaces.

The same approach described for polymer $\mathbf{3}$ was then applied to a matrix of 9 metals and 14 different polymers and the results are recorded in Figure 1a. Black crosses in the matrix signify lack of inhibition as indicated by presence of characteristic polymer signals in the IRRAS spectra of the corresponding polymers deposited on these metal surfaces. In contrast, inhibition of CVD polymerization is indicated by check marks. The significance of blue check marks as shown for polymers $\mathbf{1}$ and $\mathbf{2}$ is that these inhibitors have previously been found to prevent polymer deposition by Jensen and Vaeth ${ }^{[29]}$ and were confirmed in this study. Specifically, $\mathrm{Ag}, \mathrm{Ni}, \mathrm{Cu}$, and

(a)

(c)

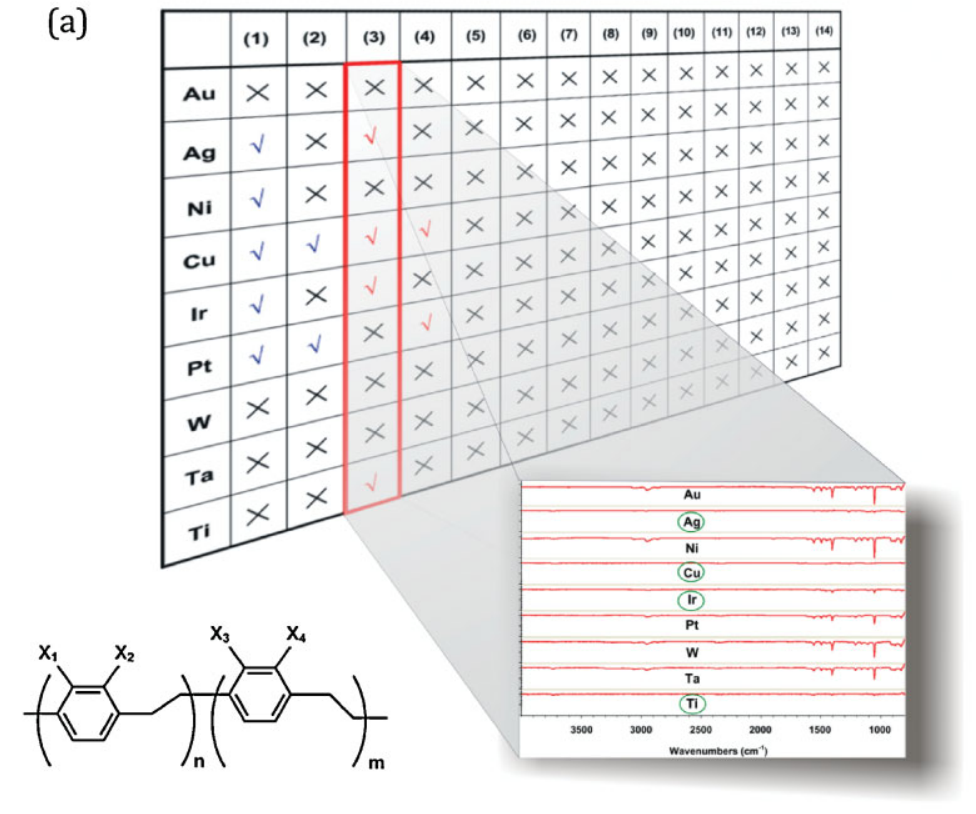

(b)
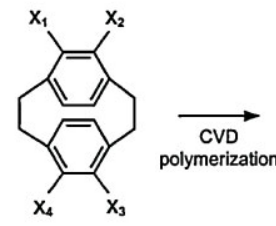

(1) (2) (3)

$\begin{array}{lllll}\text { (4) } & \text { (5) } & \text { (6) } & \text { (7) } & \text { (8) }\end{array}$

(8) (9)

(10)

$\begin{array}{cccc}(11) & (12) & (13) & (14) \\ \mathrm{COOC}_{6} \mathrm{~F}_{5} & & \mathrm{NH}_{2} & \mathrm{CH}_{2} \mathrm{NH}_{2} \\ \mathrm{H} & (\mathrm{CO})_{2} \mathrm{O} & & \mathrm{H} \\ \mathrm{H} & & & \mathrm{H} \\ \mathrm{H} & (\mathrm{CO})_{2} \mathrm{O} & \mathrm{H} & \mathrm{H}\end{array}$

Figure 1. a) Inhibition behavior of nine different metals ( $\mathrm{Au}, \mathrm{Ag}, \mathrm{Ni}, \mathrm{Cu}, \mathrm{Ir}, \mathrm{Pt}, \mathrm{W}$, Ta, and Ti) for poly-p-xylylenes deposited via CVD polymerization. b) Typical spectra for poly(dichloro-p-xylylene) (3) on $\mathrm{Au}, \mathrm{Ag}, \mathrm{Ni}, \mathrm{Cu}, \mathrm{Ir}, \mathrm{Pt}, \mathrm{W}, \mathrm{Ta}$, and Ti. The spectra are dominated by characteristic $\mathrm{C}-\mathrm{Cl}$ stretches at 1030-1100 $\mathrm{cm}^{-1}$, which were present on $\mathrm{Au}, \mathrm{Ni}, \mathrm{Pt}, \mathrm{W}$, and Ta surfaces, but not on Ag, Cu, Ir, or Ti surfaces. c) Schematic illustration shows the CVD polymerization process of [2.2]paracyclophanes that yields nonreactive (1-4) as well as reactive (5-14) poly-p-xylylenes. 
$\mathrm{Pt}$ were found to inhibit polymer $\mathbf{1}$, and $\mathrm{Cu}$ as well as $\mathrm{Pt}$ inhibited polymer 2. Iron was not included in our study, because we did not have access to an e-beam deposition system that was commissioned for iron coatings. The red check marks indicate selective inhibition that has been established for the first time in this study. For certain metals, selective CVD polymerization of polymers $\mathbf{3}$ and $\mathbf{4}$, was also observed. However, these polymers are structurally similar to polymer coatings $\mathbf{1}$ and $\mathbf{2}$, which have previously been shown by Jensen and Vaeth ${ }^{[29]}$ to undergo selective deposition. Most importantly, none of the polymers $\mathbf{1}-\mathbf{4}$ were reactive in the sense that they could support further covalent immobilization without extensive chemical alteration. In contrast, no inhibitor was found for reactive polymer coatings 5-14, which do carry functional side groups necessary for further surface chemistry. This finding suggests attractive interactions between oxygen or nitrogen atoms of the functionalized $p$-xylylenes and the metal substrates, which will result in decreased desorption of functionalized $p$-xylylenes. For a given monomer delivery rate, decreased desorption means that the critical polymer concentration will be reached faster and no inhibition may be observed in these cases. ${ }^{[29]}$ Increasing the substrate temperature should improve inhibition during CVD polymerization, ${ }^{[29]}$ but is only feasible within a relatively small range, as it also decreases deposition efficiencies. Based on our initial screening, several trends were identified: (i) selective growth was only observed on poly- $p$-xylylenes with either no substituent (1, parylene $\mathrm{N}$ ) or halogen substitutions (2-4); (ii) all poly$p$-xylylenes with either nitrogen- or oxygen-containing functional groups were found to be nonselective; (iii) the range of metals that can undergo selective inhibition is surprisingly diverse and inhibitors are quite different even for similar poly- $p$-xylylenes.

In light of these outcomes and considering the fact that the only additional polymer that has been reported to undergo selective CVD is PVP, ${ }^{[28]}$ we hypothesized that a vinylsubstituted poly- $p$-xylylene may be a promising candidate for selective deposition because of the absence of oxygen or nitrogen, its structural similarity to PV, and the fact that vinyl groups have sufficiently high reactivity to support further surface modification via cross-metathesis reactions. ${ }^{[49]}$

Prior tovapor deposition of a vinyl-substituted poly- $p$-xylylene, synthesis of the starting material, 4-vinyl[2.2]paracyclophane (15a), was conducted according to a previously reported route. ${ }^{[50]}$ Paracyclophane 15a was then subjected to CVD polymerization. Optimized CVD polymerization conditions included sublimation of $15 \mathbf{a}(10.6 \mathrm{mg})$ at $105^{\circ} \mathrm{C}$ under reduced pressure $(0.5 \mathrm{mbar})$ and subsequent transfer into the pyrolysis zone, which was maintained at $670^{\circ} \mathrm{C}$. Deposition of the polymer occurred on a temperaturecontrolled sample holder at $25^{\circ} \mathrm{C}$ with a deposition rate of $0.1 \AA \mathrm{s}^{-1}$, as determined by QCM. Under these conditions, film thicknesses were around $2-5 \mathrm{~nm}$ based on ellipsometry recorded for Si surfaces. To evaluate the adhesion properties of the as-deposited polymer $\mathbf{1 5}$, a Scotch tape test ${ }^{[23]}$ was performed to show consistent adhesion to silicon, gold, and glass substrates.
Chemical analysis of as-deposited polymer films using a combination of X-ray photoelectron spectroscopy (XPS) and IRRAS were used to confirm the proposed structure of poly(4-vinyl- $p$-xylylene-co- $p$-xylylene) (15). The IRRAS spectra reveal characteristic bands of at 2866, 2924, and $3013 \mathrm{~cm}^{-1}$ indicative of aliphatic methylene groups $(C-H)$, and the absorption peaks at 1571,1608 , and $1696 \mathrm{~cm}^{-1}$ are responsible for the aromatic vinyl $(C=C)$ stretches vibration $^{[51,52]}$ (Fig. S1 of the Supporting Information). The XPS survey spectrum (Fig. S3 of the Supporting Information) allows for quantitative evaluation of the chemical composition of polymer 15 , and the data indicate 96.9 at \% carbon relative to a theoretical value of 100 at \% obtained based on the structure of starting material 15a and assuming perfect conversion. In addition, 3.1 at \% oxygen were detected, which may be attributed to ambient contaminations or may be the result of partial oxidation. ${ }^{[24,38,43]}$ The high-resolution $\mathrm{C}_{1 \mathrm{~s}}$ spectrum (Fig. S4 of the Supporting Information), after curve fitting of the $\mathrm{C}_{1 \mathrm{~s}}$ envelope further revealed characteristic signals for $\mathrm{C}-\mathrm{C}$ and $\mathrm{C}-\mathrm{H}$ normalized to $285.0 \mathrm{eV}$ at $88.7 \%$ atomic concentration. This value compares well with the theoretical concentration of $89 \%$. Similarly, the $C=C$ signal at $286.6 \mathrm{eV}$ shows $10.0 \%$ atomic concentration, which is in good accordance with theoretical calculations. A signal indicating $\pi-\pi^{*}$ transitions at $291.5 \mathrm{eV}$ can also be found, which is characteristic for aromatic molecules and has been previously reported for similar poly- $p$-xylylenes. ${ }^{[38,43]}$ No indication of carbon in higher oxidation states (carbonyl, carboxyl) is present in the high resolution $\mathrm{C}_{1 \mathrm{~s}}$ spectrum, as it would be expected for oxidized polymer films. The excellent accordance of the high resolution $\mathrm{C}_{1 \mathrm{~s}}$ spectrum with the expected polymer structure suggests that the oxygen detected in the survey is most likely due to slight surface contaminations rather than actual oxidation of the polymer films. These findings are in accordance with our earlier work regarding the CVD polymerization of substituted [2.2]paracyclophanes, which often showed closed to theoretical compositions of the resulting functionalized poly- $p$-xylylenes. ${ }^{[38]}$ Next, we examined the stability of the polymer patterns by immersing them in a range of different solvents, including methanol, ethanol, acetone, and studying their chemical structure via IRRAS. Within the margins of error, IRRAS spectra recorded before and after solvent exposure were close to identical suggesting solvent stability of polymer $\mathbf{1 5}$ in these solvents. While the resulting polymer films were stable in aqueous solutions and certain organic solvents, such as ethanol and acetone, they dissolved in chloroform.

The ability of polymer $\mathbf{1 5}$ to undergo cross-metathesis reaction appears critical to this work, as it establishes the reactive character of the polymer coating. To clearly distinguish polymer 15 from coatings 1 to 4 with respect to their abilities to support covalent immobilization, polymer $\mathbf{1 5}$ was homogeneously deposited on the Au substrate followed by cross-metathesis reaction of OEGMA. The cross-metathesis reaction was restricted to selected areas using a microstencil placed on the substrate surface after CVD polymerization. The 
(a)

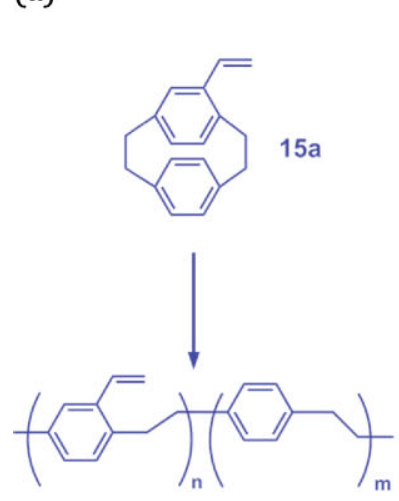

15


(b)

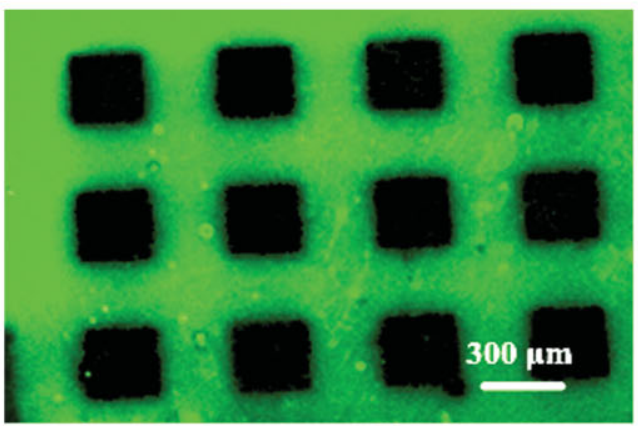

Figure 2. a) Schematic illustration shows the procedure used to demonstrate the chemical activity of polymer 15 and the characteristic properties of the subsequently modified PEGMA film. Polymer 15 was homogeneously deposited on Au followed by an olefin cross-metathesis reaction of PEGMA on a selected area through a microstencil placed on the substrate surface during CVD polymerization, resulting in $300 \mu \mathrm{m} \times 300 \mu \mathrm{m}$ of PEGMA. Fluorescence-labeled BSA was subsequently adsorbed onto the patterned surfaces. b) Fluorescence image, which reveals that strong fluorescence only occurs in areas that were not modified via the cross-metathesis reaction demonstrating that the PEGMA effectively reduces protein adsorption.

physical masking procedure resulted in a sample with $300 \mu \mathrm{m} \times 300 \mu \mathrm{m}$ of OEGMA and a background of polymer 15, as illustrated in Figure 2a. Next, fluorescence-labeled bovine serum albumin (BSA) was allowed to adsorb to the patterned surfaces. BSA was used as a model protein, because it is has a strong tendency to adsorb to hydrophobic polymer substrates. ${ }^{[24]}$ Figure $2 b$ reveals strong fluorescence signals only in areas that were not modified via cross-metathesis reaction. 


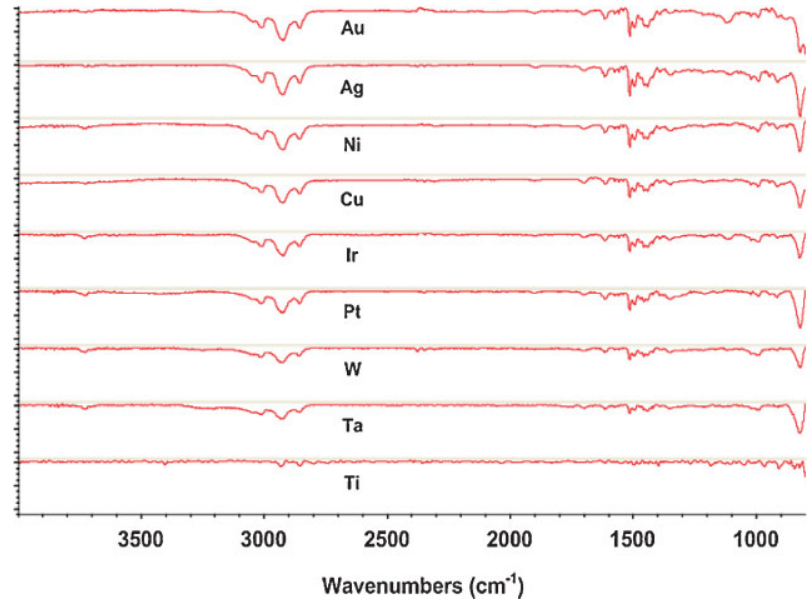

Figure 3. IRRAS spectra of poly(4-vinyl-p-xylylene-co-p-xylylene) (15) deposited on nine different metals. The result reveals $\mathrm{Ti}$ to be the only metal that inhibits the growth of 15 during CVD polymerization.

This unambiguously demonstrates the ability of polymer $\mathbf{1 5}$ to undergo surface modification via cross-metathesis reaction.

With both the synthesis of polymer $\mathbf{1 5}$ and its ability to support cross-metathesis reaction established, we then addressed the key question of this study, i.e., whether deposition of polymer $\mathbf{1 5}$ can be selectively inhibited by metals. Figure 3 summarized the IRRAS spectra of poly(4vinyl- $p$-xylylene-co-p-xylylene) (15) deposited on nine different metals (same group as studied previously). Based on the IRRAS spectra, Ti appears to be the only metal that effectively inhibits the growth of $\mathbf{1 5}$ during CVD polymerization process. Interestingly, $\mathrm{Ti}$ also inhibited CVD polymerization of polymer 3. Following the mechanism for metal-mediated inhibition of CVD polymerization proposed by Jensen and Vaeth, ${ }^{[2]}$ the metal inhibitor, Ti in this case, deactivates the $p$-xylylene and/or 4-vinyl-p-xylylene allowing only for slow accumulation on the Ti surface. In contrast, rapid polymerization occurs on the other metals resulting in significantly thicker polymer films. Although not observed under the conditions selected in this study, Jensen's study suggests the existence of an upper limit, i.e., a critical concentration of $p$-xylylenes, at which deposition will commence and relative selectivity of $\mathrm{Ti}$ will be lost. ${ }^{[29]}$ Therefore, lower monomer delivery rates and higher substrate temperatures, which, in turn, result in higher desorption rates of $p$-xylylenes form the metal surface, both favor selective deposition.

Next, we prepared a substrate, which contained circles of $\mathrm{Au}$ in a Ti background by depositing Au through a shadow mask onto a Ti-coated silicon wafer. Following the protocol outlined in Figure 4a, this bi-metal surface was CVD coated with polymer $\mathbf{1 5}$ and subsequently subjected to olefin crossmetathesis reaction with fluorescein $O$-methacrylate. As shown in Figure $4 \mathrm{~b}$, only the Au islands showed appreciable signals of fluorescence. In addition, a strong contrast can be observed between $\mathrm{Au}$ and the $\mathrm{Ti}$ surfaces providing further clarification on the selective inhibition of the deposition of polymer 15. Moreover, Ti or Au samples were coated with polymer 15 and cross-metathesis reaction of poly(ethylene glycol) methyl ether methacrylate (PEGMA) was conducted on both samples. IRRAS spectra were recorded for each modification step (Fig. S2 of the Supporting Information). In the case of the Au surface, absorption bands at 2866, 2924, and $3013 \mathrm{~cm}^{-1}$ due to $\mathrm{C}-\mathrm{H}$ symmetric and asymmetric stretching bands can be clearly detected after deposition of polymer 15 . In addition, a strong, sharp band at $1717 \mathrm{~cm}^{-1}$ indicative of the $C=O$ bond of the ester group, and a strong band at $1110 \mathrm{~cm}^{-1}$, which is due to $\mathrm{C}-\mathrm{O}-\mathrm{C}$ stretches of the ester group appeared after olefin cross-metathesis reaction of OEGMA. The contact angle changed from $89^{\circ} \pm 4^{\circ}$ before cross-metathesis reaction to $53^{\circ} \pm 4^{\circ}$ thereafter, which can be attributed to the increased hydrophilicity of OEGMA. On the other hand, no clear FT-IR signals of polymer $\mathbf{1 5}$ or OEGMA were detected on $\mathrm{Ti}$ at any of the modification steps (Fig. S2b of the Supporting Information), providing strong evidence of the inhibitory character of Ti.

In summary, we report a novel reactive polymer coating, that is, poly(4-vinyl-p-xylylene-co-p-xylylene), that can react with methacrylates via cross-metathesis reaction while being selectively inhibited by the presence of Ti. The result is the first selectively deposited, reactive CVD coating. These findings may contribute to the development of simple patterning processes that rely on selective inhibition of polymer films, rather than the use of physical aids. While it is likely that the inhibitory mechanism is similar to what has been proposed for unfunctionalized poly- $p$-xylylenes, ${ }^{[28,29]}$ future work is necessary to elucidate details of the inhibitory mechanism that leads to the selective CVD polymerization.

\section{Experimental}

Substrate Preparation: Metal substrates were fabricated from a 4-inch silicon wafer with a $3500 \AA$ insulating layer of silicon nitride followed by metal layer of $1000 \AA$. Metals of $\mathrm{Cu}$, Ir, Ta, W, and Pt were prepared by using a sputter coater (EnerJet), while $\mathrm{Ni}, \mathrm{Ti}, \mathrm{Ag}$, and $\mathrm{Au}$ were prepared by using e-beam evaporators (Cooke, and SJ-20). Patterned $\mathrm{Au}$ and $\mathrm{Ti}$ were fabricated via successive deposition using a thermal evaporator (306 vacuum evaporator, BOC/Edwards). Ti wire was purchased from Alfa Aesar, while Au pellets were purchased from Kurt J. Lesker Company. Initially, Ti (400 A) was homogeneously deposited onto silicon substrates. Next, a steel shadow mask was used to pattern subsequent $\mathrm{Au}$ layers $(200 \AA)$ ) onto the resulting Ti layers.

CVD Polymerization: Polymers 1-14 were prepared via a CVD polymerization process, and are described elsewhere [20-26, 38, 43]. Poly(4-vinyl- $p$-xylylene-co-p-xylylene) (15) was synthesized via CVD polymerization. The starting material, 4-vinyl[2.2]paracyclophane (15a) was synthesized following previously reported route [50]. The resulting 15a $(10.6 \mathrm{mg})$ was sublimed under vacuum and converted by pyrolysis into the corresponding quinodimethanes, which spontaneously polymerized upon condensation to the substrate surface. A constant argon flow of $20 \mathrm{sccm}$ was used as the carrier. Sublimation temperatures were kept at $105^{\circ} \mathrm{C}$ followed by pyrolysis at $670{ }^{\circ} \mathrm{C}$. Subsequently, polymerization occurred on a rotating sample holder maintained at $15 \pm 5^{\circ} \mathrm{C}$. Rotation of the sample holder ensured a uniform deposition of polymer, while the walls of the deposition 
(a)
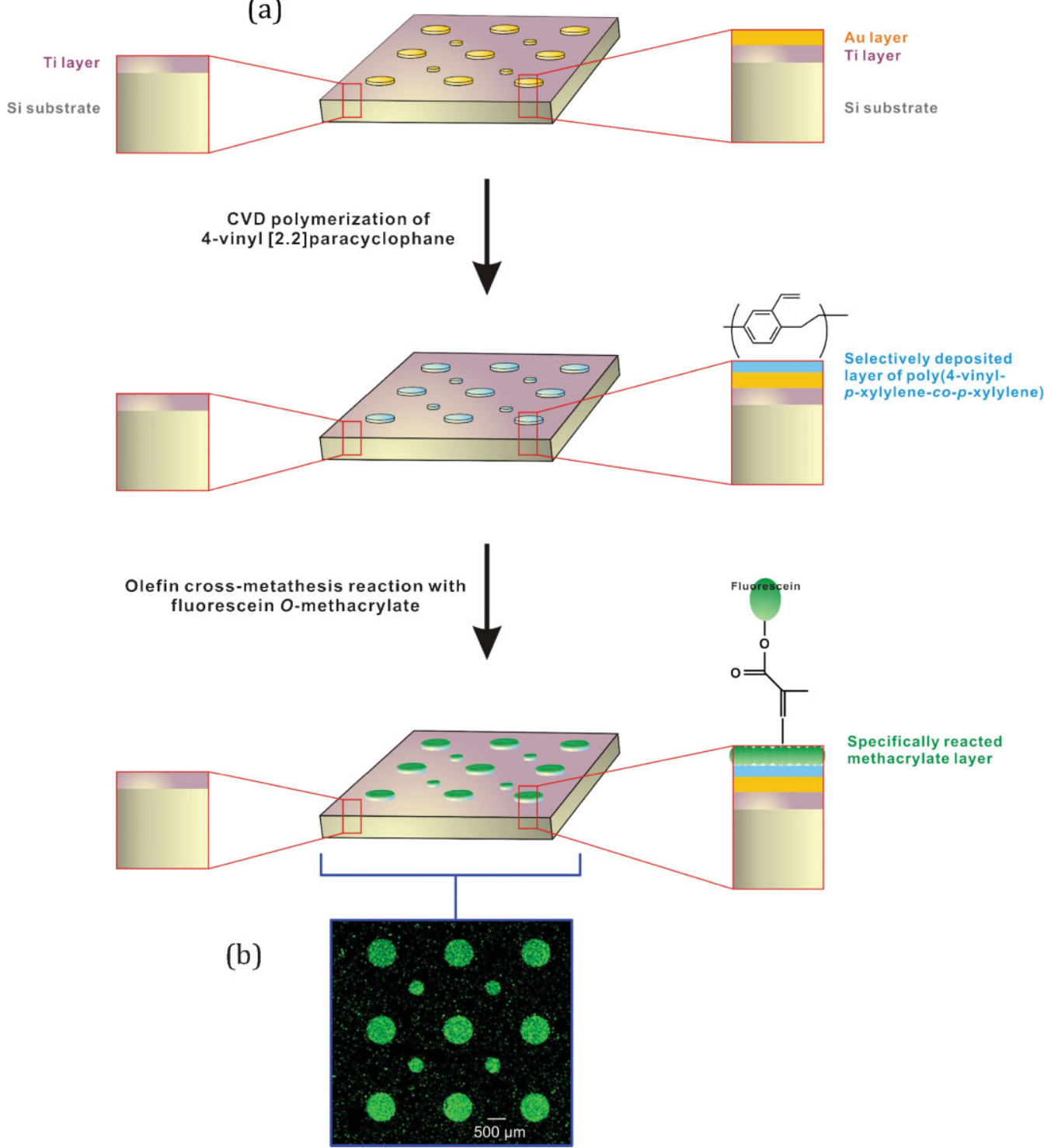

Figure 4. a) Schematic illustration of the selective deposition of polymer 15 on patterned Ti/Au substrates. Au was deposited through a shadow mask onto a Ti-coated silicon wafer followed by polymer 15 deposition via CVD polymerization. Spatially controlled surface modification of terminal vinyl groups of polymer 15 via olefin cross-metathesis reaction with fluorescein O-methacrylate confirmed selective CVD of a functionalized poly-p-xylylene. b) Fluorescence image, which reveals that only the Au islands showed appreciable signals of fluorescence. Scale bar is $200 \mu \mathrm{m}$.

chamber was maintained at $120^{\circ} \mathrm{C}$ to prevent any residual deposition on the chamber walls. The coating pressure was $0.5 \mathrm{mbar}$ or below.

Surface Characterizations: XPS data were recorded on an Axis Ultra X-ray photoelectron spectrometer (Kratos Analyticals, UK) equipped with a monochromatized $\mathrm{Al} \mathrm{K} \alpha \mathrm{X}$-ray source. In these experiments, the lens mode was in hybrid, X-ray power is $150 \mathrm{~kW}$, and pass energy was set to $160.0 \mathrm{eV}$ for the survey acquisition and $20.0 \mathrm{eV}$ for high resolution C1s acquisition. Thickness analysis was recorded using a single wavelength $(532 \mathrm{~nm}) \mathrm{EP}^{3}$-SW imaging ellipsometry (Nanofilm Technologie GmbH, Germany). Four zone nulling experiments were performed at an angle of incidence of $72^{\circ}$, and a constant $n$ (refractive index) and $k$ (extinction coefficient) value model was used to model the ellipsometric parameters psi and delta. IR spectroscopy was performed on a Nicolet 6700 spectrometer to detect key functional groups characteristic of the polymer. The grazing angle accessory (Smart SAGA) was utilized at a grazing angle of $85^{\circ}$.
Surface Reaction: Polymer 15 was deposited via CVD polymerization onto silicon substrates, and surface patterns were created by using a microstencil during CVD process. The deposited polymer films were reacted with PEGMA (MW 475, Sigma-Aldrich) via an olefin cross-methathesis reaction [39] catalyzed by ruthenium-based Grubbs catalyst (second generation, Sigma-Aldrich). In a reaction vial containing the coated substrates, PEGMA $(0.25 \mathrm{~mL}, 0.57 \mathrm{mmol})$ dissolved in ethanol $(0.75 \mathrm{~mL})$ was added along with Grubbs catalyst $(1.875 \mathrm{mg}, 0.0022 \mathrm{mmol})$. The reaction vial was capped and then placed in a heat bath maintained at $50^{\circ} \mathrm{C}$ for $4 \mathrm{~h}$. After reaction, the substrates were removed, washed three times with about $1.5 \mathrm{~mL}$ of ethanol per rinse, and then dried. For olefin cross-metathesis reaction with selectively deposited polymer $\mathbf{1 5}$ on the patterned Au-Ti surfaces, fluorescein $O$-methacrylate (Sigma-Aldrich) catalyzed by Grubbs catalyst was used to react with the deposited films. In a reaction vial containing the coated substrates, fluorescein $O$-methacrylate $(76 \mathrm{mg}$, 
$0.19 \mathrm{mmol})$ and Grubbs catalyst $(1.875 \mathrm{mg}, 0.0022 \mathrm{mmol})$ were dissolved in ethanol $(1 \mathrm{~mL})$. The reaction vial was capped and then placed in a heat bath maintained at $50^{\circ} \mathrm{C}$ for $4 \mathrm{~h}$. After reaction, the substrates were removed, washed three times with ethanol, and then dried.

Protein Adsorption: Rhodamine-conjugated BSA (Invitrogen/ Molecular Probes) was used as model protein to study the fouling property of PEGMA-modified surfaces. Protein solutions were prepared at a concentration of $58 \mu \mathrm{g} / \mathrm{mL}$. Samples were incubated with the protein solution for $10 \mathrm{~min}$. After incubation, samples were thoroughly rinsed with PBS containing $0.1 \% \mathrm{w} / \mathrm{v}$ BSA and Tween 20 $(0.02 \% \mathrm{v} / \mathrm{v})$. The resulting samples were then examined by using a GenePix 4000B scanner with $532 \mathrm{~nm}(17 \mathrm{~mW})$ at a gain of 400 GMT. The excitation wavelength was scanned at $10 \mu \mathrm{m}$ spatial resolution.

Received: February 16, 2008 Revised: March 31, 2008 Published online: August 5, 2008

[1] J. D. Tian, H. Gong, N. J. Sheng, X. C. Zhou, E. Gulari, X. L. Gao, G. Church, Nature 2004, 432, 1050 .

[2] J. P. Pellois, X. C. Zhou, O. Srivannavit, T. C. Zhou, E. Gulari, X. L. Gao, Nat. Biotechnol. 2002, 20, 922.

[3] A. Berg, W. Olthuis, P. Bergveld, Micro Total Analysis Systems 2000, Kluwer Academic, Norwell, MA 2000.

[4] A. Y. Fu, C. Spence, A. Scherer, F. H. Arnold, S. R. Quake, Nat. Biotechnol. 1999, 17, 1109 .

[5] C. S. Chen, M. Mrksich, S. Huang, G. M. Whitesides, D. E. Ingber, Science 1997, 276, 1425 .

[6] H. Gu, C. Xu, L.-T. Weng, B. Xu, J. Am. Chem. Soc. 2003, 125, 9256.

[7] H. W. Gu, R. K. Zheng, X. X. Zhang, B. Xu, Adv. Mater. 2004, 16, 1356.

[8] O. Ambacher, J. Phys. D. Appl. Phys. 1998, 31, 2653.

[9] Y. Kashima, T. Munakata, A. Matoba, Opt. Rev. 1997, 4, 69.

[10] T. F. Kuech, Semicond. Sci. Technol. 1993, 8, 967.

[11] D. Dendukuri, D. C. Pregibon, J. Collins, T. A. Hatton, P. S. Doyle, Nat. Mater. 2006, 5, 365 .

[12] H. Y. Chen, J. M. Rouillard, E. Gulari, J. Lahann, Proc. Natl. Acad. Sci. USA 2007, 104, 11173.

[13] R. Langer, D. A. Tirrell, Nature 2004, 428, 487.

[14] R. S. Tu, M. Tirrell, Adv. Drug Deliv. Rev. 2004, 56, 1537.

[15] C. J. Hawker, K. L. Wooley, Science 2005, 309, 1200.

[16] M. Yoshida, R. Langer, A. Lendlein, J. Lahann, Polym. Rev. 2006, 46, 347.

[17] Y. V. Pan, R. A. Wesley, R. Luginbuhl, D. D. Denton, B. D. Ratner, Biomacromolecules 2001, 2, 32.

[18] N. A. Alcantar, E. S. Aydil, J. N. Israelachvili, J. Biomed. Mater. Res. 2000, $51,343$.

[19] L. Tang, C. Tsai, W. W. Gerberich, L. Kruckeberg, D. R. Kania, Biomaterials 1995, 16, 483.
[20] J. Lahann, Polym. Int. 2006, 55, 1361.

[21] J. Lahann, M. Balcells, H. Lu, T. Rodon, K. F. Jensen, R. Langer, Anal. Chem. 2003, 75, 2117.

[22] J. Lahann, D. Klee, W. Pluester, H. Hoecker, Biomaterials 2001, 22, 817.

[23] H. Nandivada, H. Y. Chen, J. Lahann, Macromol. Rapid Commun. 2005, 26, 1794

[24] H. Y. Chen, J. Lahann, Anal. Chem. 2005, 77, 6909.

[25] J. Lahann, I. S. Choi, J. Lee, K. F. Jensen, R. Langer, Angew. Chem. Int. Ed. 2001, 40, 3166.

[26] J. Lahann, M. Balcells, T. Rodon, J. Lee, I. S. Choi, K. F. Jensen, R. Langer, Langmuir 2002, 18, 3632.

[27] H. Y. Chen, J. Lahann, Adv. Mater. 2007, 19, 3801.

[28] K. M. Vaeth, K. F. Jensen, Adv. Mater. 1999, 11, 814.

[29] K. M. Vaeth, K. F. Jensen, Chem. Mater. 2000, 12, 1305.

[30] K. Y. Suh, R. Langer, J. Lahann, Appl. Phys. Lett. 2003, 83, 4250.

[31] J. J. Senkevich, S. B. Desu, Thin Solid Films 1998, 322, 148.

[32] T. P. Martin, S. E. Kooi, S. H. Chang, K. L. Sedransk, K. K. Gleason, Biomaterials 2007, 28, 909.

[33] S. J. Limb, C. B. Labelle, K. K. Gleason, D. J. Edell, E. F. Gleason, Appl. Phys. Lett. 1996, 68, 2810.

[34] N. H. Lee, C. W. Frank, Langmuir 2003, 19, 1295.

[35] Y. C. Chang, C. W. Frank, Langmuir 1998, 14, 326.

[36] P. Hanefeld, U. Westedt, R. Wombacher, T. Kissel, A. Schaper, J. H. Wendorff, A. Greiner, Biomacromolecules 2006, 7, 2086.

[37] H. W. Gu, C. J. Xu, L. T. Weng, B. Xu, J. Am. Chem. Soc. 2003, 125, 9256.

[38] J. Lahann, R. Langer, Macromolecules 2002, 35, 4380

[39] J. Lahann, H. Hocker, R. Langer, Angew. Chem. Int. Ed. 2001, 40, 726.

[40] J. Lahann, D. Klee, H. Hocker, Macromol. Rapid Commun. 1998, 19 , 441.

[41] J. Lahann, R. Langer, Macromol. Rapid Commun. 2001, 22, 968.

[42] X. Jiang, H. Y. Chen, G. Galvan, M. Yoshida, J. Lahann, Adv. Funct. Mater. 2008, 18, 27.

[43] H. Nandivada, H. Y. Chen, L. Bondarenko, J. Lahann, Angew. Chem. Int. Ed. 2006, 45, 3360.

[44] Y. Elkasabi, H. Y. Chen, J. Lahann, Adv. Mater. 2006, 18, 1521.

[45] D. K. Peng, S. T. Yu, D. J. Alberts, J. Lahann, Langmuir 2007, 23, 297.

[46] Y. S. Shon, E. Cutler, Langmuir 2004, 20, 6626.

[47] R. Voicu, A. Badia, F. Morin, R. B. Lennox, T. H. Ellis, Chem. Mater. 2001, 13, 2266.

[48] P. E. Laibinis, G. M. Whitesides, D. L. Allara, Y. T. Tao, A. N. Parikh, R. G. Nuzzo, J. Am. Chem. Soc. 1991, 113, 7152.

[49] J. A. Love, M. S. Sanford, M. W. Day, R. H. Grubbs, J. Am. Chem. Soc. 2003, $125,10103$.

[50] S. Iwatsuki, T. Itoh, M. Kubo, H. Okuno, Polym. Bull. 1994, $32,27$.

[51] M. A. Gauthier, I. Stangel, T. H. Ellis, X. X. Zhu, Biomaterials 2005, 26, 6440 .

[52] J. S. Major, G. J. Blanchard, Chem. Mater. 2002, 14, 2567. 